\title{
Microlensing of pulsar-radiation
}

\author{
Norbert Wex \\ Max Planck Society, Research Unit "Theory of Gravitation", at the \\ University of Jena, Max-Wien-Platz 1, D-07743 Jena, Germany \\ Janusz Gil and Marek Sendyk \\ Astronomical Centre, Pedagogical University, \\ Lubuska 2, 65-265 Zielona Góra, Poland
}

\section{Pulsar-Timing and Microlensing}

When the radiation of a star passes near a massive object, its intensity is magnified due to the microlensing phenomenon. Similar effects should also apply to pulsar signals. However, because of the pulsating nature of pulsar radiation, the flux magnification will be accompanied by a time varying travel-time delay (Krauss and Small 1991, Larchenkova and Doroshenko 1995, Ohnishi et al. 1995, Wex et al. 1996).

Let us denote the epoch of observation by $t_{\oplus}$. A travel-time delay caused by a Schwarzschild-lens is characterized by four parameters: $M$, the mass of the lens, $q$, the ratio of the transverse velocity of the lens with respect to the line of sight and the minimum impact parameter, $f_{m}$, the ratio of the minimum impact parameter and the Einstein radius, and $T_{0}$, the time of maximum delay. If we denote the "intrinsic" frequency and its derivative with respect to time (corresponding to the barycentric arrival time $t=t_{\oplus}$ ) by $\nu$ and $\dot{\nu}$ then we find for the observed phase of the pulsar at the barycentric time $e^{2} t$ :

$$
\phi=\phi_{0}+\nu\left(t-t_{\oplus}\right)+\frac{1}{2} \dot{\nu}\left(t-t_{\oplus}\right)^{2}+\nu \tau_{M}\left(4 F_{ \pm}^{-2}-2 \ln F_{ \pm}\right)
$$

where

$$
\tau_{M}=\frac{2 G M}{c^{3}}, \quad F_{ \pm}=\sqrt{f^{2}+4} \pm f, \quad f=f_{m} \sqrt{1+q^{2}\left(t-T_{0}\right)^{2}} .
$$

(+ denotes the brighter and - the fainter of the two signals).

In case of a weak lensing event $\left(f_{m} \gg 1\right)$ only the strong $(+)$ signal is observable and the last term in Eq. (1) simplifies to the expression of the usual Shapiro delay without the parameter $f_{m}$

To get the expected accuracies for the determination of the parameters $(M, q, \ldots)$ we performed Monte Carlo simulations to generate synthetic data sets. Assuming bi-weekly timing-observations for a period of 10 years with $1 \mu \mathrm{s}$ timing-accuracy, $v_{\perp}=300 \mathrm{~km} / \mathrm{s}, T_{0}-t_{0}=5$ yr and $f_{m} \gg 1$, we find:

\footnotetext{
${ }^{2}$ Note: "intrinsic" denotes the frequency and its derivatives measured in the absence of any lensing mass.
} 


\begin{tabular}{l|cccc}
\hline \hline$M\left[M_{\odot}\right]$ & 0.1 & 1 & 10 & 20 \\
$b_{m}[\mathrm{AU}]$ & - & 10 & 50 & 100 \\
\hline$\Delta M / M$ & - & $10 \%$ & $5 \%$ & $7 \%$ \\
$\Delta q / q$ & - & $20 \%$ & $5 \%$ & $5 \%$ \\
$\Delta\left(t_{\oplus}-T_{0}\right) /\left(t_{\oplus}-T_{0}\right)$ & - & $2 \%$ & $0.5 \%$ & $0.5 \%$ \\
\hline$b_{m}^{*}[\mathrm{AU}]$ & 20 & 150 & 350 & 500 \\
\hline
\end{tabular}

where $b_{m}^{*}$ denotes the maximum $b_{m}$ at which the microlensing event would still show up in the residuals.

For lenses below $0.5 M_{\odot}$ the parameter determination is rather poor, thus microlensing of pulsar radiation cannot be used for the mass-determination of small stellar objects, e.g. brown dwarfs.

\section{Probability for microlensing events for pulsars in the Galactic Center Region}

The expected number $\mathcal{N}$ of stars between the pulsar at a distance $D$ and the Earth which are able to influence noticeably the time of arrival of the observed pulsar signals is given by:

$$
\mathcal{N}=\int_{0}^{D} \int_{0.08}^{\infty} \pi\left[b_{m}^{*}(\mathcal{M})\right]^{2} \xi(\mathcal{M}, l) d \mathcal{M} d l,
$$

where $l$ parameterizes the line of sight and $\xi(\mathcal{M}, r)$ gives the number density of the stars that have a mass between $\mathcal{M}\left(\mathcal{M} \equiv M\left[M_{\odot}\right]\right.$ ) and $\mathcal{M}+d \mathcal{M}$ (see Bahcall and Soneira 1980, Kroupa 1995). For $b_{m}^{*}$ one finds after extensive Monte Carlo simulations

$$
b_{m}^{*}[\mathrm{AU}] \approx \begin{cases}250 \mathcal{M}^{1.1} & \text { if } 0.1 \leq \mathcal{M}<0.5 \\ 150 \mathcal{M}^{0.4} & \text { if } 0.5 \leq \mathcal{M}<20 .\end{cases}
$$

(cf. previous table). For a pulsar situated $0.5 \mathrm{kpc}$ behind the Galactic Center and at an angular distance $\theta$ from the Galactic Center, as seen on the sky, one obtains:

\begin{tabular}{l|cccc}
\hline \hline$\theta[$ deg & 2.0 & 1.0 & 0.5 & 0.1 \\
\hline $\mathcal{N}$ & 0.03 & 0.08 & 0.15 & 0.5 \\
\hline
\end{tabular}

\section{References}

Bahcall, J.N., Soneira, R.M., 1980, ApJS, 44, 73

Krauss, L.M., Small, T.A., 1991, ApJ, 378, 22

Kroupa, P., 1995, ApJ, 453, 358

Larchenkova, T.I., Doroshenko, 1995, A\&A, 297, L607

Ohnishi, K., Hosokawa, M., Fukushima, T., Takeuti, M., 1995, ApJ, 448, 2710

Wex, N., Gil, J., Sendyk, M., 1996, A\&A, in press, astro-ph/9511023 